\title{
Combined Blood Indexes of Systemic Inflammation as a Mirror to Admission to Intensive Care Unit in COVID-19 Patients: A Multicentric Study
}

\author{
Dina Ali Hamad'1 ${ }^{1}$. Mai Mostafa Aly ${ }^{2}$. Marwa Ahmed Abdelhameid ${ }^{3}$. Shimaa Abdalla Ahmed ${ }^{4}$ \\ Asmaa Salah Shaltout ${ }^{5}$. Alaa Eldin Abdel-Moniem ${ }^{1} \cdot$ Ahmed Mohamed Reda Ragheb $^{6} \cdot$ Mohammed Nahed Attia $^{7}$. \\ Taghreed Sayed Meshref ${ }^{1}$
}

Received: 9 September 2021 / Accepted: 29 November 2021 / Published online: 13 December 2021

(c) The Author(s) 2021

\begin{abstract}
Background The Coronavirus 2019 is a pandemic that has spread worldwide, threatening human health. The main cause of death in patients with COVID-19 is a systemic pro-inflammatory mechanism that quickly progresses to acute respiratory distress syndrome. Hematological ratios as affordable indicators of inflammatory response were studied in COVID-19 patients. The study aimed to study the importance of the blood cell indexes of the systemic inflammatory response, as the Aggregate Index of Systemic Inflammation (AISI), neutrophils lymphocyte to platelet ratio (NLPR), systemic immuneinflammation index (SII) and, systemic inflammation response index (SIRI) in predicting intensive care unit (ICU) admission of COVID-19 patients.

Methods 495 COVID-19 patients managed in four tertiary centers; divided into non-ICU and ICU groups.

Results Total leucocyte count (TLC), AISI, NLPR, SII, and SIRI were more elevated in the ICU group $(P<0.001$ for all except AMC $P=0.006)$, while this group had less absolute lymphocyte count (ALC) $(P=0.047)$.

We estimated the optimal cut-off values of the hematological ratio; AISI (729), NLPR (0.0195), SII (1346), and SIRI (2.5). SII had the highest specificity (95.6\%), while NLPR had the highest sensitivity (61.3\%). Age, AISI, CRP, D-dimer, and oxygen aid were the independent predictors for ICU admission in COVID-19 in multivariate logistic regression.

Conclusion AISI is a predictor for severity and ICU admission in COVID-19 patients, SII is a predictor of survival, while NLPR and SIRI have an additive role that needs further evaluation.
\end{abstract}

Keywords COVID-19 $\cdot$ AISI $\cdot$ SII $\cdot$ Intensive care unit $\cdot$ Egypt

$\begin{array}{ll}\text { Abbreviations } \\ \text { AISI } & \text { Aggregate Index of Systemic Inflammation } \\ \text { CBC } & \text { Complete blood picture } \\ \text { COPD } & \text { Chronic obstructive pulmonary disease } \\ \text { CPAP } & \text { Continuous positive ventilation pressure } \\ \text { CRP } & \text { C-reactive protein } \\ \text { CT } & \text { Computed tomography } \\ \text { CVD } & \text { Cardiovascular disease } \\ \text { DM } & \text { Diabetes mellitus } \\ \text { HTN } & \text { Hypertension } \\ \text { ICU } & \text { Intensive care unit } \\ \text { NLPR } & \text { Neutrophils lymphocyte to platelet ratio }\end{array}$

Dina Ali Hamad

dinaalihamad@aun.edu.eg

Extended author information available on the last page of the article
NLR Neutrophil to lymphocyte ratio

LMR Lymphocyte to monocyte ratio

PLR Platelet to lymphocyte ratio

SII Systemic immune-inflammation index

SIRI Systemic inflammation response index

WHO World Health Organization

\section{Introduction}

COVID-19 continued to spread, invading about 223 countries worldwide [1]. Epidemiologists believe COVID-19 is here to stay, and annual fluctuations in infection could exist by 2025 and beyond [2]. Low- and middle-income countries are expected to suffer longer than other countries, probably as the future of this virus is affected by social and economic status. Almost $20 \%$ of hospitalized patients need ICU 
admission, with a mortality rate reaching $61.5 \%$ in some regions [3]. Also, COVID-19 patients admitted to ICU have a twofold greater risk of thrombotic complications than nonICU patients [4].These facts force us to investigate cheap and informative methods to detect the risky population who may need intensive care unit (ICU) admission.

Blood indexes were extensively studied in COVID-19 patients, such as the neutrophil to lymphocyte ratio (NLR) [5], derived NLR [5], platelet to lymphocyte ratio (PLR) [6], and lymphocyte to monocyte ratio (LMR) [7]. Red cell distribution width (RDW) was another important blood index that was studied in COVID-19 patients. A previous meta-analysis showed that elevated RDW is associated with adverse outcomes in COVID-19 patients [8].Other indexes, which include three or more blood values, are less studied with COVID-19, such as Aggregate Index of Systemic Inflammation (AISI), neutrophils lymphocyte to platelet ratio (NLPR), systemic immune-inflammation index (SII) and, systemic inflammation response index (SIRI).

AISI (neutrophils*monocytes* platelets/lymphocytes) is a unique parameter in neoplastic conditions as non-smallcell lung cancer [9]. Very few studies evaluated its link to COVID-19 victims; none of them was linked to severity [10]. Among thousands of researches on COVID-19, only one detected the predictive value of NLPR (neutrophil to lymphocyte*platelet ratio) in infected patients [11].

SII, which depends on the number of lymphocytes, neutrophils, and platelets, is an indicator associated with inflammation and can reflect the immune and inflammatory state [12]. Some studies have identified a clear correlation between SII and prognosis in malignancy and inflammatory conditions [13, 14]. However, there are limited data about the benefit of SII in assessing the prognosis in COVID-19 patients.

It is observed that SIRI (neutrophil*platelet to lymphocyte ratio) is correlated with clinical outcomes and predicts the survival of gastric [15] and breast cancers [16]. This efficient parameter can properly represent the inflammatory and immune status balance with datasets in COVID-19 patients [10].

In this research, we aimed to study these combined blood cell indexes of systemic inflammation, the association between AISI, NLPR, SII, and SIRI and the need for ICU admission hoping to contribute to clinical practice.

\section{Methods}

\subsection{Study Design}

This retrospective study included 495 COVID-19 patients admitted in four tertiary hospitals in Egypt (Assiut University Hospital, El Rajhi Hospital, Aswan University Hospital,
Qena University Hospital) during July 2020. COVID19 diagnosis based on WHO interim guidelines [17]. All patients were treated according to recommendations from the World Health Organization (WHO) [17].

\subsection{Data Collection}

Data of the patients were collected from the hospital records following the patients' consents to share the data and the authorization of the local research ethics committee according to the Declaration of Helsinki. It included age, sex, history of smoking, associated comorbidities [diabetes mellitus (DM), hypertension (HTN), cardiovascular disease (CVD), chronic obstructive pulmonary disease (COPD)], vital signs at admission, duration of hospitalization, treatment (steroid and oxygen supply either by low flow oxygen supplementation (nasal cannula, facial masks, or non-rebreather facial masks) or high-flow oxygen supplementation (highflow nasal cannula, continuous positive ventilation pressure (CPAP) or mechanical ventilation), and outcomes.

Investigations included complete blood picture (CBC), $\mathrm{d}$-dimer, ferritin and $\mathrm{C}$ reactive protein (CRP). Blood Indexes of Systemic Inflammation were calculated from CBC according to the following equations: AISI $=$ neutrophil*platelet* monocyte to lymphocyte ratio, NLPR = neutrophil to lymphocyte* platelet ratio, SII = neutrophil*platelet to lymphocyte ratio and, SIRI = neutrophil* monocyte to lymphocyte ratio. All collected laboratory results were recorded at day of admission.

Computed tomography (CT) of the chest of the patients was classified into specific findings suggestive of COVID19 infection as bilateral or unilateral multifocal ground-glass opacities that classically predominate in the peripheral, posterior, and basal part of the lungs or other less specific findings. CORADS classification was scored from very low or CO-RADS 1 to very high or CO-RADS 6 based on the CT findings.

Patients were grouped according to admission site into (1) ICU group: patients with severe presentations who were admitted to ICU. (2) Non- ICU group: patients with less severe presentations admitted to the ward. The decision regarding ICU admission depended on the Modified National Early Warning Score (Modified NEWS) for COVID-19 patients [18]. Supplementary Table 1.

\subsection{Statistical Analysis}

Continuous variables were either expressed as suitable means and standard deviations or medians and interquartile ranges. Categorical variables were presented as the counts and percentages in each category. We grouped the patients into ICU and non-ICU groups. Wilcoxon rank-sum analysis was employed to continuous variables, and for categorical 
variables, chi-square and Fisher's exact tests were used. By applying the receiver operating curve (ROC) analysis, we determined the optimal cut-off values of the continuous AISI, NLPR, SII, and SIRI. Kaplan-Meier curves of AISI, NLPR, SII, and SIRI were used to determine the survival time of COVID-19 patients. As common indicators to assess relative risk, hazard risk (HR) and 95\% confidence interval (CI) were used. Binary logistic regression analysis was performed to assess the effect of age, gender, and all other relevant factors. $P<0.05$ was considered to be statistically significant. All these data analyses were conducted with the software SPSS 170 (SPSS Inc, Chicago, USA).

\section{Results}

\subsection{Clinical, Laboratory, and Imaging Characteristics of the Studied Population}

Demographic and baseline data of the studied cohort are shown in (Table 1$)$. This study populations were divided into two groups: non-ICU $(n=185 ; 37.4 \%)$ and ICU $(n=310$; $62.6 \%$ ). Older patients were more in the ICU group in comparison to the non-ICU group (median $=58$ vs. 33 years) $(P<0.001)$. Most of the patients were males in both groups $(P=0.41)$.

Regarding the associated comorbidities, most ICU patients had D.M., HTN, CVD, and COPD compared to the non-ICU group $(P<0.001)$.

Regarding the hematological parameters, hemoglobin level was higher in the non-ICU group vs. ICU group, with a significant difference $(P=0.021)$. On the other side, platelets (PLT) were lower in the non-ICU group $(P<0.001)$.

The total leucocyte count (TLC), absolute neutrophil count (ANC), and, NLR were higher in the ICU group with a statistically significant difference $(P<0.001)$. Absolute monocyte count (AMC) was also statistically significantly higher in the ICU group $(P=0.006)$. In comparison, absolute lymphocyte count (ALC) was lower in the ICU than in the non-ICU group, with a significant difference $(P=0.047)$.

The AISI, NLPR, SII, and SIRI were higher in the ICU group with statistically significant difference $(P<0.001)$.

Inflammatory markers in our study showed that higher CRP and d-dimer were found in the ICU group $(P<0.001)$, while more elevated ferritin tended to be in the non-ICU group $(P=0.175)$.

MSCT imaging showed more extensive lesions (either bilateral lesions or multiple unilateral lesions) in all patients of the ICU group compared to $90.8 \%$ of the non-ICU group $(P<0.001)$.

The oxygen aid, either low flow as simple facial masks, nasal cannula or non-rebreather facial masks or high flow as continuous positive ventilation pressure (CPAP), was given to $87.1 \%$ of ICU group patients compared to $14.6 \%$ of nonICU group $(P<0.001)$. One-third $(36.1 \%)$ of ICU patients were mechanically ventilated.

Death occurred in $7 \%$ vs. $38.7 \%$ in non-ICU and ICU groups, respectively, with a significant difference between both groups $(\mathrm{P}<0.001)$.

\subsection{ROC Curves to Detect the Optimum Cut-Off Values of Hematological Indexes to Differentiate ICU from Non-ICU COVID-19 Infection}

We analyzed the optimal cut-off values of AISI, NLPR, SII, and SIRI calculated by the ROC analysis and presented in (Fig. 1). The area under the curve (AUC) of AISI, NLPR, SII, and SIRI was $0.807,0.768,0.819$, and 0.815 . The optimal cut-off values were $728,0.0195,1346$, and 2.5 for AISI, NLPR, SII, and SIRI, respectively. SII had the highest specificity (95.6\%) followed by AISI, then SIRI, then NLPR $(92.8 \%, 91.9 \%$, and $80.9 \%$ respectively), while the highest sensitivity was in favor of NLPR (61.3), then SIRI, then AISI, and last SII $(59.4 \%, 51.7 \%, 50.9 \%$ respectively) (Table 2).

\subsection{Kaplan-Meier Curves of AISI, NLPR, SII, and SIRI to Determine the Survival Time of COVID-19 Patients}

The estimated mean time until death was 21.35 days for nonICU, and 17.75 days for the ICU group $(P<0.001)$.

Figure 2 shows that the survival probability is lower for ICU patients at all-time points, so they are less likely to survive.

Mean survival time was estimated according to AISI (estimated mean time until death is 22.1 days for victims with AISI $<729$ and 17.9 days for those with AISI $>729$, NLPR (The estimated mean time until death is 21.93 days for patients with NLPR $<0.0195$ and 17.12 days for patients with NLPR $>0.0195$ ), SII (the estimated mean time until death is 20.96 days if SII $<1346$ and 16.82 days if SII $>1346$ ), and SIRI (The estimated mean time until death is 21.43 days if SIRI $<2.5$ and 17.89 days if SIRI $>2.5$ ). So, it is obvious that those patients with AISI $>729$, NLPR $>0.0195$, SII $>1346$, and SIRI $>2.5$ are less likely to survive (Fig. 3).

\subsection{Identification of Possible Predictors of ICU Admission in COVID-19 Cases}

To determine the impact of the above indicators on patients' prognosis with COVID-19, we also performed Kaplan-Meier survival analysis and COX regression analysis to explore the possible independent predictors for ICU 
Table 1 Demographic data of the studied population

\begin{tabular}{|c|c|c|c|}
\hline \multirow[t]{2}{*}{ Variant } & \multicolumn{2}{|l|}{ Group } & \multirow[t]{2}{*}{$P$-value } \\
\hline & $\begin{array}{l}\text { Non-ICU } \\
n=185\end{array}$ & $\begin{array}{l}\mathrm{CCU} \\
n=310\end{array}$ & \\
\hline Age/years(median) & 33 & 58 & $<0.001 *$ \\
\hline Sex /males $n(\%)$ & $91(49.2 \%)$ & $181(58.4 \%)$ & $0.013 * *$ \\
\hline Smoking $n(\%)$ & $37(20.0 \%)$ & $65(21.0 \%)$ & $0.754 * *$ \\
\hline \multicolumn{4}{|l|}{ Comorbidities $n(\%)$} \\
\hline $\mathrm{DM}$ & $11(6.0 \%)$ & $81(26.1 \%)$ & $<0.001 * *$ \\
\hline HTN & $14(7.6 \%)$ & $113(36.5 \%)$ & $<0.001 * *$ \\
\hline CVD & $9(4.9 \%)$ & $85(27.4 \%)$ & $<0.001 * *$ \\
\hline COPD & $5(2.7 \%)$ & $35(11.3 \%)$ & $<0.001 * * *$ \\
\hline \multicolumn{4}{|l|}{ Laboratory data $($ mean $\pm S D)$} \\
\hline $\mathrm{HB}(\mathrm{g} / \mathrm{dL})$ & $12.14 \pm 2.54$ & $11.67 \pm 2.55$ & $0.021 *$ \\
\hline Platelets $\left(\times 10^{9} / \mathrm{L}\right)$ & $208.03 \pm 103.65$ & $256.55 \pm 130.87$ & $<0.001 *$ \\
\hline $\mathrm{TLC}\left(\times 10^{9} / \mathrm{L}\right)$ & $5.31 \pm 3.57$ & $10.79 \pm 7.06$ & $<0.001 *$ \\
\hline $\mathrm{ANC}\left(\times 10^{9} / \mathrm{L}\right)$ & $2.96 \pm 2.55$ & $8.40 \pm 5.88$ & $<0.001 *$ \\
\hline $\operatorname{ALC}\left(\times 10^{9} / \mathrm{L}\right)$ & $1.72 \pm 1.22$ & $1.56 \pm 1.06$ & $0.047 *$ \\
\hline $\mathrm{AMC}\left(\times 10^{9} / \mathrm{L}\right)$ & $0.41 \pm 0.44$ & $0.69 \pm 0.91$ & $0.006 *$ \\
\hline NLR & $2.04 \pm 1.68$ & $7.40 \pm 6.61$ & $<0.001 *$ \\
\hline AISI & $256.38 \pm 505.47$ & $1318.81 \pm 1948.85$ & $<0.001 *$ \\
\hline NLPR & $0.01 \pm 0.02$ & $0.04 \pm 0.05$ & $<0.001 *$ \\
\hline SII & $492.29 \pm 804.49$ & $2016.29 \pm 2162.88$ & $<0.001 *$ \\
\hline SIRI & $1.05 \pm 1.60$ & $4.91 \pm 7.60$ & $<0.001 *$ \\
\hline $\mathrm{CRP}(\mathrm{mg} / \mathrm{l})$ & $33.30 \pm 44.74$ & $96.82 \pm 120.29$ & $<0.001 *$ \\
\hline Ferritin $(\mathrm{mcg} / \mathrm{ml})$ & $241.74 \pm 221.54$ & $235.55 \pm 237.43$ & 0.175* \\
\hline D-dimer (mcg/ml) & $0.63 \pm 0.46$ & $4.57 \pm 2.92$ & $<0.001 *$ \\
\hline \multicolumn{4}{|l|}{$\operatorname{MSCT} n(\%)$} \\
\hline $\begin{array}{l}\text { Bilateral affection or multiple } \\
\text { unilateral affection }\end{array}$ & $168(90.8 \%)$ & $310(100.0 \%)$ & $<0.001 * * *$ \\
\hline \multicolumn{4}{|l|}{ Oxygen support $n(\%)$} \\
\hline $\begin{array}{l}\text { Any oxygen aids other than } \\
\text { mechanical ventilation }\end{array}$ & $27(14.6 \%)$ & $112(36.1 \%)$ & $<0.001 * *$ \\
\hline Mechanical ventilation & $0(0.0 \%)$ & $99(67.8)$ & $<0.001 * *$ \\
\hline \multicolumn{4}{|l|}{ Outcome $n(\%)$} \\
\hline Death & $13(7 \%)$ & $120(38.7 \%)$ & $<0.001 * *$ \\
\hline
\end{tabular}

Bold indicates $P$ value $<0.05$ are statistically significant

AISI Aggregate Index of Systemic Inflammation, ALC absolute lymphocytic count, ANC absolute monocyte count, $A N C$ absolute neutrophil count, $C O P D$ chronic obstructive pulmonary disease, $C R P$ C-reactive protein, $C V D$ Cardio Vascular Disease, DM diabetes mellitus, $H B$ hemoglobin, $H T N$ hypertension, $L M R$ lymphocyte to monocyte ratio, $M S C T$ multi-slice computed tomography, $N L P R$ neutrophils lymphocyte to platelet ratio, $N L R$ neutrophil to lymphocyte ratio, SII systemic immune-inflammation index, SIRI systemic inflammation response index, WBCs white blood cells

*Mann-Whitney test

*Chi-square test

***Fisher`s Exact test

admission of COVID-19. Further univariate and multivariate $\mathrm{COX}$ regression analysis showed that AISI(HR $1.000,95 \%$ CI 1.000-1.002), NLPR(HR 1.647, 95\% CI 0.280-9.681), SII(HR 1.001, 95\% CI 1.001-1.003) and SIRI (HR 1.015, 95\% CI 1.003-1.027) were identified by univariate Cox regression but only raised SII(HR 1.004,
95\% CI 1.000-1.006) was the independent factor affecting the recovery and discharge of patients with COVID-19 in multivariate analysis.

Age, male sex, DM, HTN, cardiovascular diseases, COPD, NLR, AISI, NLPR, SII, SIRI, CRP, d-dimer, steroid, oxygen aids, and mechanical ventilation were consistent 


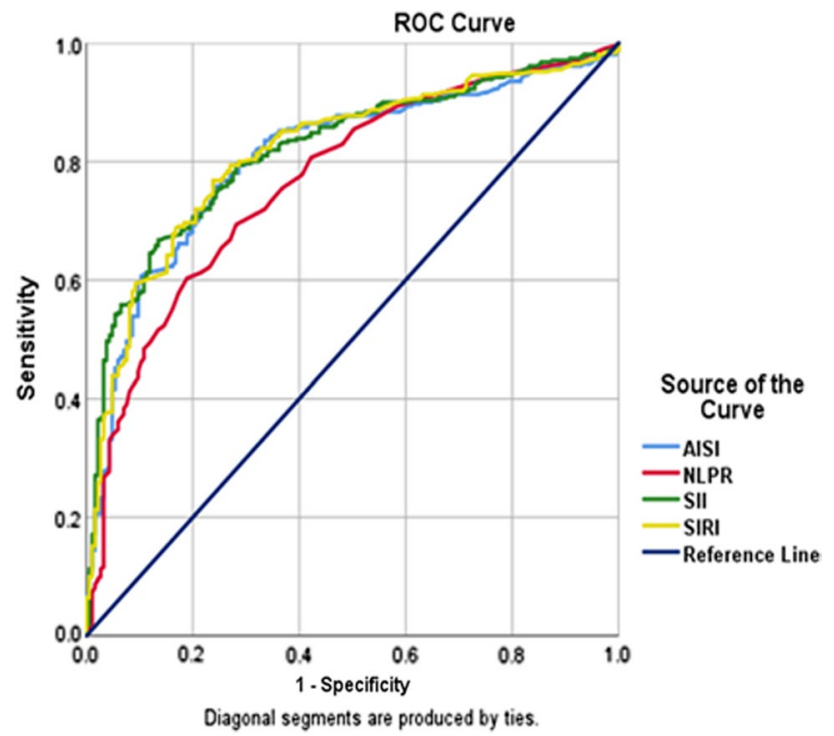

Fig. 1 ROC curves to detect the optimum cut-off values of hematological ratios, AISI; Aggregate Index of Systemic Inflammation (>729); NLPR; neutrophils lymphocyte to platelet ratio (>0.0195); SII; systemic immune-inflammation index $(>1346)$ and SIRI; systemic inflammation response index $(>2.5)$

with COVID-19 disease severity in the univariate logistic regression study. We integrated all the above parameters with statistical significance in the univariate analysis for in-depth analysis into the multivariate logistic regression model. In the multivariate logistic regression model, considering the likelihood of overfitting, we assumed a stepwise forward method for logistic regression analysis to decrease the number of independent variables entering the model, aiming to decrease the probability of overfitting the model. The results showed that the early independent predictors for ICU entry in COVID-19 were age, AISI, CRP, D-dimer, and oxygen support following admission (Table 3 ).

As indicated by multivariable analysis, the three biomarkers (AISI, CRP, and D-dimer) were independent predictors for ICU admission. The area under ROC curve of the combination of these three parameters was 0.98. The predictive ability of joint indicators showed the superiority over the single index $(P<0.001)$ (Fig. 4$)$.

\section{Discussion}

COVID-19 is the most lethal pandemic of recent history, with a large spectrum of severity, ranging from mild or even asymptomatic cases to severe presentations requiring ICU admission. COVID-19 infection showed fluctuations in the disease spread, with peaks exceeding half a million cases per day worldwide, so ICU capacities are insufficient to face such increasing demands. Consequently, we used data obtained at the peak of COVID-19 in 2020, aiming to assist in the ICU admission decision depending on easily accessible, rapid, and simple tests such as CBC.

Older patients often have more comorbidities and less immunity, so it is well accepted to have a higher risk for admission in ICU. Like our study, many other studies proved the relation between COVID 19 severity and old age [19-21]. However, a large analysis performed on more than 5000 cases did not detect a significant age difference between ICU and non-ICU patients [22].

In considering comorbidities as DM, HTN, COPD, and CVD, the ICU group in our study had significantly more associated diseases than in previous studies [20,23]. Medical history of other conditions was a top predictor of mortality in COVID 19 patients than a parameter for ICU entry in other studies [15, 24].

$\mathrm{CBC}$ has been widely used to test inflammatory processes and diagnose many diseases [25]. Being simple, inexpensive, and informative investigation, $\mathrm{CBC}$ has been extensively investigated in the majority of COVID 19 studies. Higher WBCs, ANC, AMC, and NLR were observed in more critical COVID-19 patients [10, 23, 26], and this is in line with our results. ALC and PLT predominate in less severe cases [10, 20,24]. Neutrophils are the first step of innate immune defense. They play a crucial defensive role
Table 2 Area under curve (AUC) of AISI, NLPR, SII and SIRI

\begin{tabular}{lccccccc}
\hline \multirow{2}{*}{ AUC } & $P$-value & \multicolumn{2}{l}{$95 \%$ CI } & Cut off point & Sensitivity (\%) & Specificity (\%) \\
\cline { 4 - 5 } & & & Lower & Upper & & \\
\hline AISI & 0.807 & $<\mathbf{0 . 0 0 1}$ & 0.767 & 0.846 & $>729$ & 51.7 & 91.9 \\
NLPR & 0.768 & $<\mathbf{0 . 0 0 1}$ & 0.725 & 0.810 & $>0.0195$ & 61.3 & 80.9 \\
SII & 0.819 & $<\mathbf{0 . 0 0 1}$ & 0.782 & 0.856 & $>1346$ & 50.9 & 95.6 \\
SIRI & 0.815 & $<\mathbf{0 . 0 0 1}$ & 0.777 & 0.854 & $>2.5$ & 59.4 & 92.8 \\
\hline
\end{tabular}

The test variable(s): AISI, NLPR, SII and SIRI had at least one tie between the positive actual state group and the negative actual state group.

AISI Aggregate Index of Systemic Inflammation, AUC area under curve, $C I$ confidence interval, NLPR neutrophils lymphocyte to platelet ratio, SII systemic immune-inflammation index, SIRI systemic inflammation response index, $P V$ predictive value 
Fig. 2 Survival rate using Kaplan-Meier in the studied groups; (non-ICU and ICU groups) showing mean survival 21.35 days in non-ICU vs. 17.75 days in ICU patients $(\mathrm{P}<0.001)$

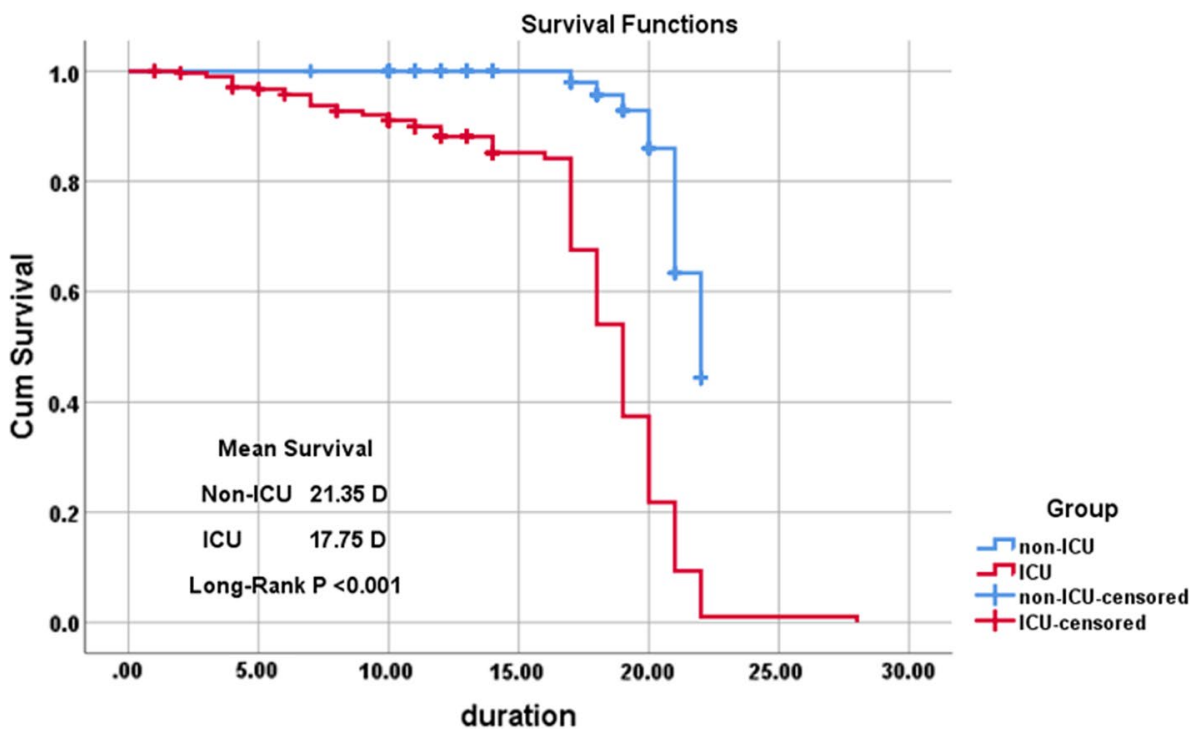

in bacterial and fungal infection by destroying these microorganisms via phagocytosis and creating neutrophil extracellular traps (NET). However, their attribute in viral infections remains unclear. Neutrophils do not seem essential for virus clearance from pulmonary cells and host survival in mice infected by SARS-CoV [27]. The autopsy of patients affected by COVID-19 demonstrated significant neutrophil infiltration in pulmonary capillaries and extravasation into alveolar spaces. The development of both trachea neutrophilic mucositis and acute capillaritis indicates profound inflammation in the airways [27]. Lymphopenia described in COVID-19, on the other hand, seems to be related to the virus's ability to infect $\mathrm{T}$ cells depending on angiotensinconverting enzyme 2 (ACE2) receptors and CD147-spike protein [28].

The combined ratios of these parameters are often used as inflammation indices. These parameters are recommended as biomarkers to assist in diagnosing inflammation, progression, and risk stratification. The NLR, derived NLR (d-NLR), PLR, and monocyte to lymphocyte ratio (MLR) have recently been proved to play a crucial role in diagnosis and severity evaluation of COVID-19 cases [18, 19, 29]; Interestingly, it has been documented that NLR value is a sensitive inflammation marker than absolute neutrophil and lymphocyte levels [28]. However, up to our knowledge, none of the previous research works have assessed the predictive value of SII, NLPR, SIRI, and AISI to assess the need of COVID-19 patients for ICU admission.

ROC curve was used to detect the optimal cut-off values of SII, NLPR, SIRI, and AISI, which revealed that SII and SIRI had the highest areas under the curve, with SII having an optimal cut-off value of 1346 and SIRI had an optimal cut-off value of 2.5. A previous study which investigated the optimal cut-off values of these indexes, detected significant
AUC with SIRI (cut-off value 2.9) and AISI (cut-off value 798), while borderline significant AUC with SII [11]. On the contrary, another study revealed NLPR to be the index with the highest AUC (0.7); AUC was in line with those of previous reports, ranging between 0.65 and 0.73 [30-32]. SII, which relies on thrombocytes, neutrophils, and lymphocytes, is a recently proposed score. The SII was suggested as a prognostic marker in sepsis patients' follow-up, as an index describing the instability in the inflammatory response [32]. Moreover, SII effectively predicts the prognosis of hepatocellular carcinoma and small cell lung cancer [12]. In another interesting study, compared to healthy controls, SII was significantly altered in COVID-19 patients, indicating a diagnostic role in patients infected with SARS-CoV2 [25].

In accordance with a previous study, Kaplan-Meier survival curves using cut-off values obtained from ROC curves showed that survival was significantly related to AISI, NLPR, SII, and SIRI [11]. Kaplan-Meier survival curves showed substantially lower survival in patients with higher AISI, NLPR, SII, and SIRI.

Univariate and multivariate logistic regression was conducted to predict possible factors that increase the need for admission to ICU; among all studied clinically factors, only age, AISI, CRP, D-dimer, and use of oxygen aid were independent early predictors for ICU admission while the role of other combined indexes as NLPR, SI, and SIRI was unclear. According to previous research, AISI was significantly higher in COVID-19 pneumonic patients than in nonpneumonic cases [9]. This coincides with our observations in which AISI was higher in the ICU group than the nonICU group as all ICU patients were pneumonic, as evidenced by MSCT imaging. In a previous similar study, elevated SII was the independent adverse factor affecting COVID 19 
A

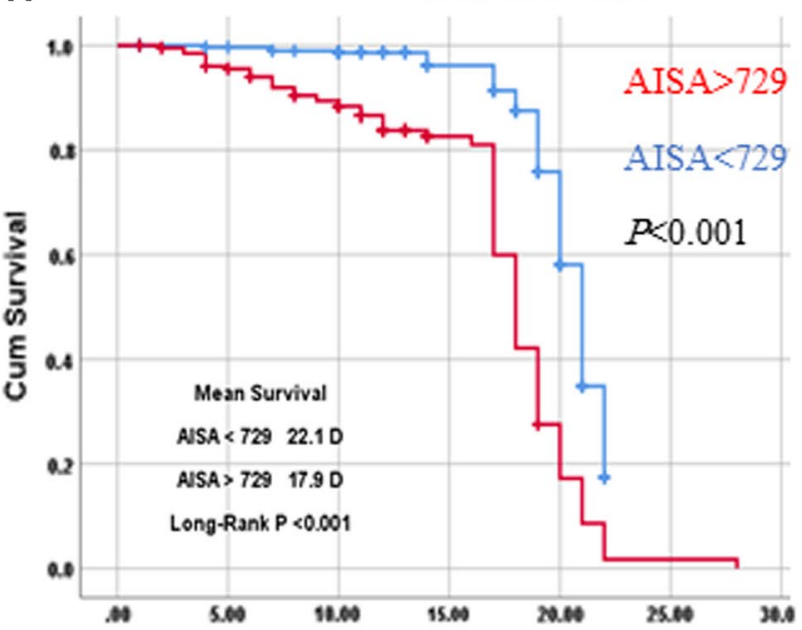

C

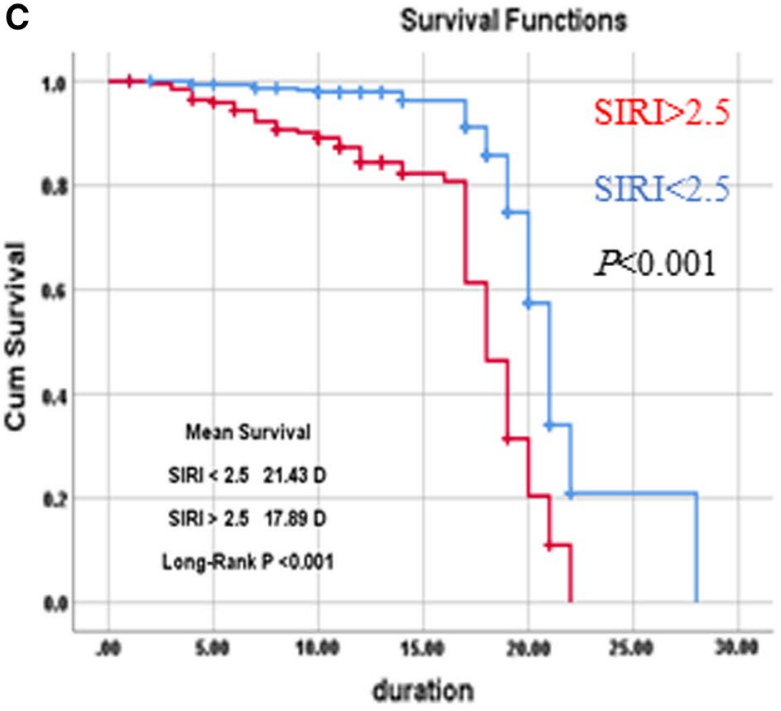

Fig. 3 Survival analysis using Kaplan-Meier curves according to the studied blood indexes of systemic inflammation: A AISI $>729$ vs AISI $<729 ; P<0.001$, B NLRP $>0.0195$ vs. NLRP $<0.0195$; $P=0.007$ C SIRI $>2,5$ vs. $\mathrm{SIRI}<2.5 ; P<0.001$ and (D)SII $>1346$

patients' survival after adjusting for confounders reported in univariate analysis [11].

The limitations of this research included its retrospective design, the complexity of the used indices which may create a difficulty to use them in the practice, and the probability of related confounders, despite attempts to prevent it. Another study limitation is that unlike other biomarkers, there has been no clear consensus on the standard cut-off values of AISI, NLPR, SII, and SIRI. It
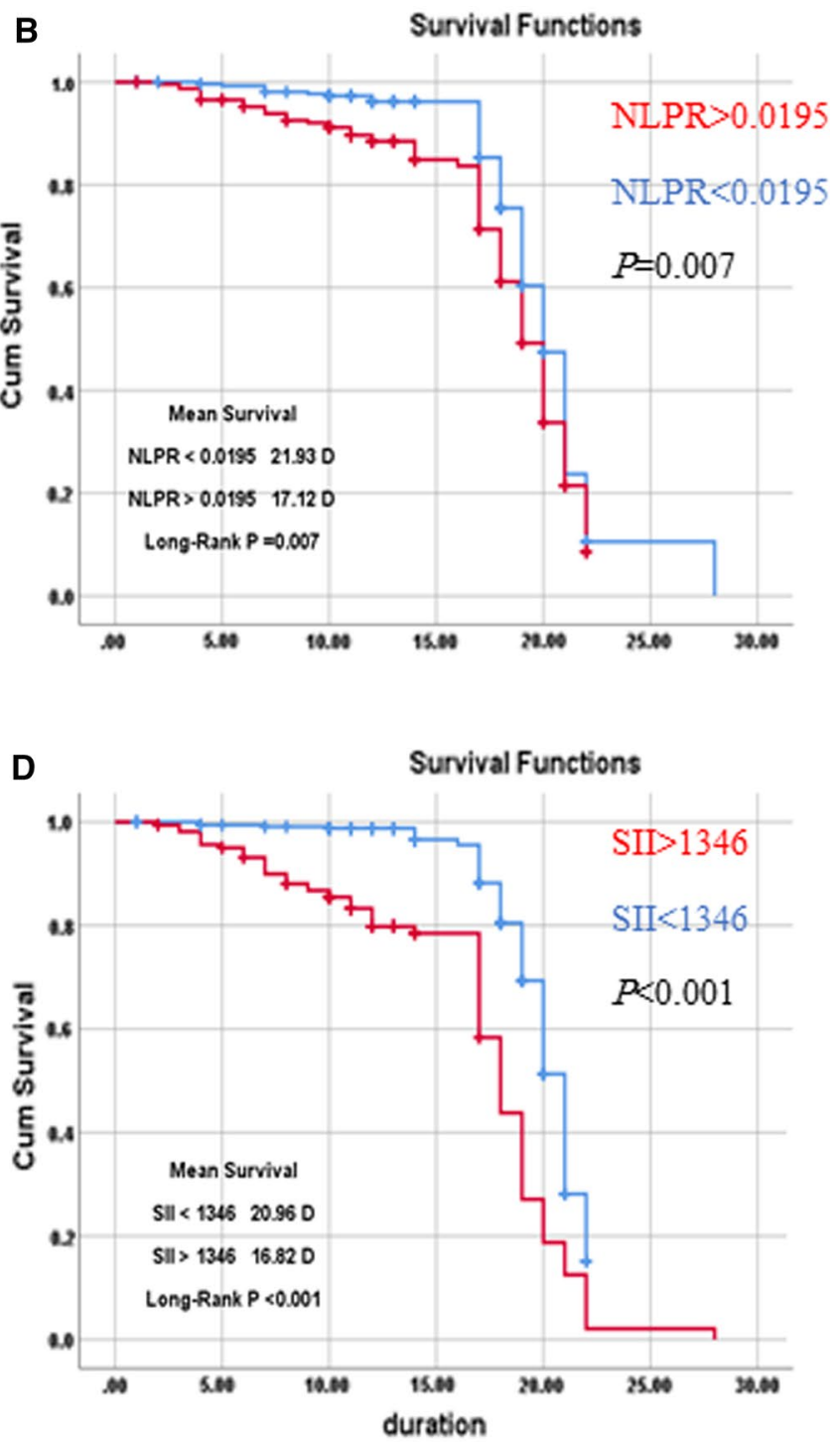

vs. SII $<1346 ; P<0.001$. AISI, Aggregate Index of Systemic Inflammation; NLPR, neutrophils lymphocyte to platelet ratio; SII, systemic immune-inflammation index; SIRI, systemic inflammation response index

is unknown whether the cutoffs identified in this study can be applied to other populations.

\section{Conclusion}

According to our results and observations in the current study, AISI appears a reasonable and attractive predictor for admission to ICU. We encourage early calculation 
Table 3 Univariate and multivariate logistic regression analysis of risk factors associated with ICU admission in COVID-1

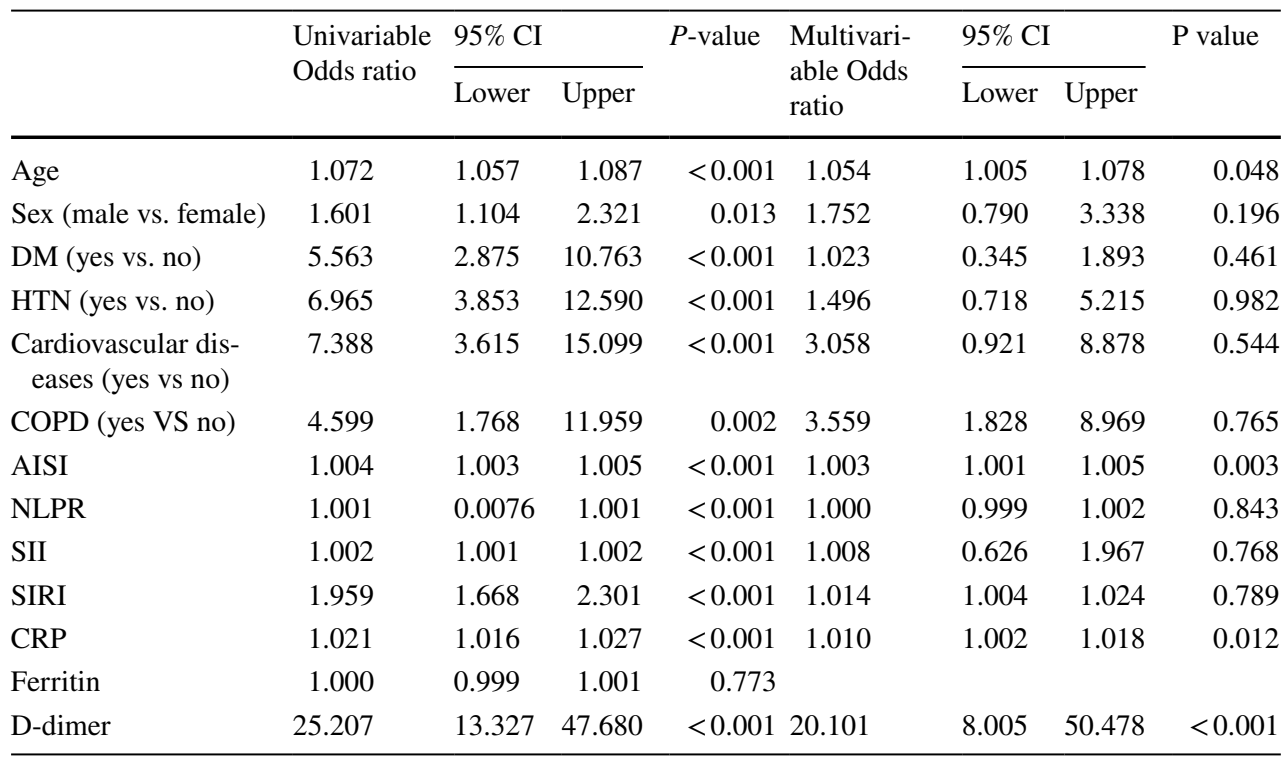

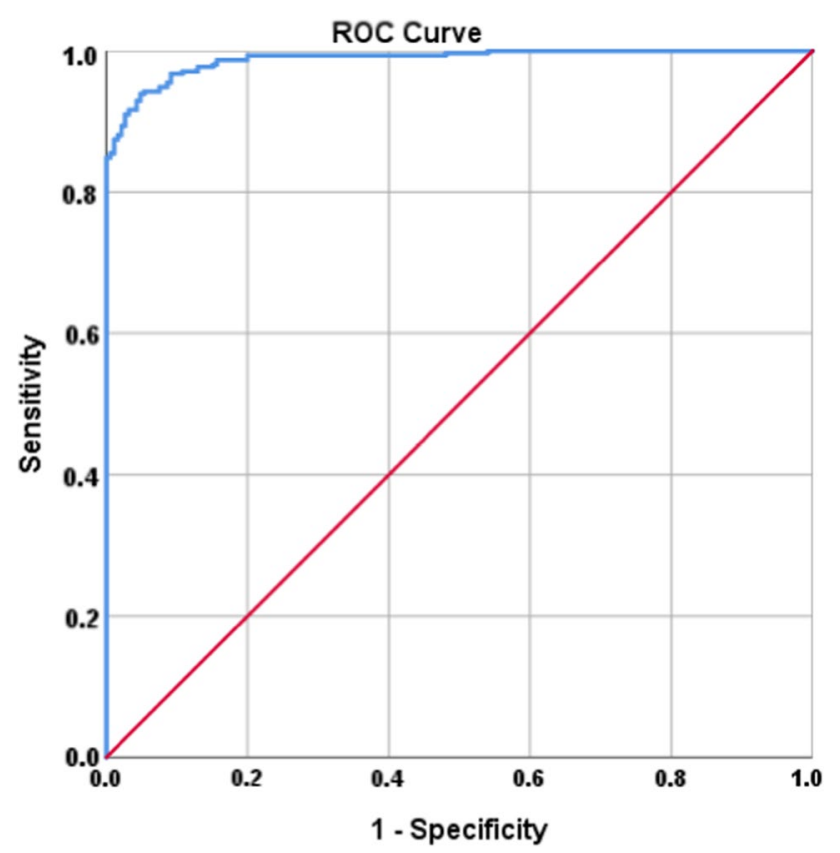

Fig. 4 ROC curve to detect the predicted probability of the joint indicators (AISI, CRP and D-dimer) for ICU admission. The area under ROC curve of the combination of these three parameters was 0.98 $(P<0.001)$. AISI Aggregate Index of Systemic Inflammation, CRP $\mathrm{C}$-reactive protein, $I C U$ intensive care unit

of this unique score to sort outpatients of COVID-19 on admission. SII was linked to survival rather than admission to ICU, while the other indexes mostly had an additive role that needed further evaluations. The use of AISI, D-dimer, and CPR together showed a predictive ability higher than the use of a single index.
AISI Aggregate Index of Systemic Inflammation, COPD chronic obstructive pulmonary disease, $C R P$ C-reactive protein, $D M$ diabetes mellitus, $H T N$ hypertension, $N L P R$ neutrophils lymphocyte to platelet ratio, SII, systemic immune-inflammation index, SIRI systemic inflammation response index

Supplementary Information The online version contains supplementary material available at https://doi.org/10.1007/s44197-021-00021-5.

Author contributions DH, TM and MA designed the study, contributed to data collection and data entry, interpreted the data and wrote the manuscript. MA, AS, AR, SA and MN collect the data and performed the statistics. AA designed the study and contributed in writing the manuscript. All authors reviewed and approved the final version of the manuscript. The corresponding author attests that all listed authors meet authorship criteria.

Funding This research did not receive any funding.

Data availability The data that support the findings of this study are available from the corresponding author upon reasonable request.

\section{Declarations}

Conflict of interest The authors declare that they have no known competing financial interests or personal relationships that could have appeared to influence the work reported in this paper.

Ethical approval Data of the patients were gathered from the hospital reports following the authorization of the local research ethics committee (IRB number: 17300513) according to the Declaration of Helsinki.

Open Access This article is licensed under a Creative Commons Attribution 4.0 International License, which permits use, sharing, adaptation, distribution and reproduction in any medium or format, as long as you give appropriate credit to the original author(s) and the source, provide a link to the Creative Commons licence, and indicate if changes 
were made. The images or other third party material in this article are included in the article's Creative Commons licence, unless indicated otherwise in a credit line to the material. If material is not included in the article's Creative Commons licence and your intended use is not permitted by statutory regulation or exceeds the permitted use, you will need to obtain permission directly from the copyright holder. To view a copy of this licence, visit http://creativecommons.org/licenses/by/4.0/.

\section{References}

1. Rahmandad H, Lim TY, Sterman J. Behavioral dynamics of COVID-19: Estimating under-reporting, multiple waves, and adherence fatigue across 92 nations. 2021;92.

2. Scudellari M. How the pandemic might play out in 2021 and beyond. Nature. 2020;584(7819):22-5.

3. Rodriguez-Morales AJ, Cardona-Ospina JA, Gutiérrez-Ocampo E, Villamizar-Peña R, Holguin-Rivera Y, Escalera-Antezana JP, et al. Clinical, laboratory and imaging features of COVID-19: a systematic review and meta-analysis. Travel Med Infect Dis. 2020;34:101623-36.

4. Chi G, Lee JJ, Jamil A, Gunnam V, Najafi H, Memar Montazerin $\mathrm{S}$, et al. Venous thromboembolism among hospitalized patients with COVID-19 undergoing thromboprophylaxis: a systematic review and meta-analysis. J Clin Med. 2020;9(8):2489.

5. Yang A-P, Liu J, Tao W, Li H-M. The diagnostic and predictive role of NLR, d-NLR and PLR in COVID-19 patients. Int Immunopharmacol. 2020;84:106504.

6. Qu R, Ling Y, Zhang YZ, Wei LY, Chen X, Li XM, et al. Plateletto-lymphocyte ratio is associated with prognosis in patients with coronavirus disease-19. J Med Virol. 2020;92(9):1533-41.

7. Merekoulias G, Alexopoulos EC, Belezos T, Panagiotopoulou E, Jelastopulu E. Lymphocyte to monocyte ratio as a screening tool for influenza. PLoS Currents. 2010. https://doi.org/10.1371/curre nts.RRN1154.

8. Lee JJ, Montazerin SM, Jamil A, Jamil U, Marszalek J, Chuang ML, et al. Association between red blood cell distribution width and mortality and severity among patients with COVID19: A systematic review and meta-analysis. J Med Virol. 2021;93(4):2513-22.

9. Paliogiannis P, Putzu C, Cortinovis D, Colonese F, Canova S, Fois A, et al. Blood cell count indexes of systemic inflammation as predictive biomarkers of immunotherapy outcomes in advanced non-small-cell lung cancer 2018.

10. Paliogiannis P, Zinellu A, Scano V, Mulas G, De Riu G, Pascale $\mathrm{RM}$, et al. Laboratory test alterations in patients with COVID-19 and non COVID-19 interstitial pneumonia: a preliminary report. J Infect Dev Count. 2020;14(07):685-90.

11. Fois AG, Paliogiannis P, Scano V, Cau S, Babudieri S, Perra $\mathrm{R}$, et al. The systemic inflammation index on admission predicts in-hospital mortality in COVID-19 patients. Molecules. 2020;25(23):5725.

12. Hu B, Yang X-R, Xu Y, Sun Y-F, Sun C, Guo W, et al. Systemic immune-inflammation index predicts prognosis of patients after curative resection for hepatocellular carcinoma. Clin Cancer Res. 2014;20(23):6212-22.

13. Li H, Huang J-b, Pan W, Zhang C-t, Chang X-y, Yang B. Systemic Immune-Inflammatory Index predicts prognosis of patients with COVID-19: a retrospective study. 2020.

14. Furuncuoğlu Y, Tulgar S, Dogan A, Cakar S, Tulgar Y, Cakiroglu B. How obesity affects the neutrophil/lymphocyte and platelet/ lymphocyte ratio, systemic immune-inflammatory index and platelet indices: a retrospective study. Eur Rev Med Pharmacol Sci. 2016;20(7):1300-6.

15. Li S, Lan X, Gao H, Li Z, Chen L, Wang W, et al. Systemic Inflammation Response Index (SIRI), cancer stem cells and survival of localised gastric adenocarcinoma after curative resection. J Cancer Res Clin Oncol. 2017;143(12):2455-68.

16. Chen L, Kong X, Wang Z, Wang X, Fang Y, Wang J. Pretreatment systemic inflammation response index in patients with breast cancer treated with neoadjuvant chemotherapy as a useful prognostic indicator. Cancer Manag Res. 2020;12:1543.

17. Organization WH. Clinical management of severe acute respiratory infection (SARI) when COVID-19 disease is suspected: interim guidance, 13 March 2020. World Health Organization; 2020.

18. Liao X, Wang B, Kang Y. Novel coronavirus infection during the 2019-2020 epidemic: preparing intensive care units-the experience in Sichuan Province, China. Intensive Care Med. 2020;46(2):357-60.

19. Guan W-J, Ni Z-Y, Hu Y, Liang W-H, Ou C-Q, He J-X, et al. Clinical characteristics of coronavirus disease 2019 in China. N Engl J Med. 2020;382(18):1708-20.

20. Zhao Z, Chen A, Hou W, Graham JM, Li H, Richman PS, et al. Prediction model and risk scores of ICU admission and mortality in COVID-19. PLoS ONE. 2020;15(7):e0236618.

21. Zhou F, Yu T, Du R, Fan G, Liu Y, Liu Z, et al. Clinical course and risk factors for mortality of adult inpatients with COVID19 in Wuhan, China: a retrospective cohort study. The lancet. 2020;395(10229):1054-62.

22. Li X, Ge P, Zhu J, Li H, Graham J, Singer A, et al. Deep learning prediction of likelihood of ICU admission and mortality in COVID-19 patients using clinical variables. PeerJ. 2020;8:e10337.

23. Qin C, Zhou L, Hu Z, Zhang S, Yang S, Tao Y, et al. Dysregulation of immune response in patients with COVID-19 in Wuhan. China. Clin Infect Dis. 2020;71(15):762-8.

24. Huang G, Kovalic AJ, Graber CJ. Prognostic value of leukocytosis and lymphopenia for coronavirus disease severity. Emerg Infect Dis. 2020;26(8):1839-41.

25. Usul E, Şan İ, Bekgöz B, Şahin A. Role of hematological parameters in COVID-19 patients in the emergency room. Biomark Med. 2020;14(13):1207-15.

26. Huang C, Wang Y, Li X, Ren L, Zhao J, Hu Y, et al. Clinical features of patients infected with 2019 novel coronavirus in Wuhan. China The lancet. 2020;395(10223):497-506.

27. Tomar B, Anders H-J, Desai J, Mulay SR. Neutrophils and neutrophil extracellular traps drive necroinflammation in COVID-19. Cells. 2020;9(6):1383.

28. Chan AS, Rout A. Use of neutrophil-to-lymphocyte and platelet-to-lymphocyte ratios in COVID-19. J Clin Med Res. 2020;12(7):448.

29. Aly MM, Meshref TS, Abdelhameid MA, Ahmed SA, Shaltout AS, Abdel-Moniem AE, et al. Can hematological ratios predict outcome of COVID-19 patients? a multicentric study. J Blood Med. 2021;12:505.

30. Tatum D, Taghavi S, Houghton A, Stover J, Toraih E, Duchesne J. Neutrophil-to-lymphocyte ratio and outcomes in Louisiana Covid19 patients. Shock (Augusta, Ga). 2020;54:652-8. https://doi.org/ 10.1097/SHK.0000000000001585.

31. Zhang H, Cao X, Kong M, Mao X, Huang L, He P, et al. Clinical and hematological characteristics of 88 patients with COVID-19. Int J Lab Hematol. 2020;42(6):780-7.

32. Lagunas-Alvarado M, Mijangos-Huesca FJ, Terán-González J, Lagunas-Alvarado MG, Martínez-Zavala N, Reyes-Franco I, et al. Systemic immune inflammatory index in sepsis. Med Intern México. 2017;33(3):303-9. 


\section{Authors and Affiliations}

\section{Dina Ali Hamad ${ }^{1}$ (1) Mai Mostafa Aly ${ }^{2}$. Marwa Ahmed Abdelhameid ${ }^{3}$. Shimaa Abdalla Ahmed ${ }^{4}$ Asmaa Salah Shaltout ${ }^{5}$. Alaa Eldin Abdel-Moniem ${ }^{1} \cdot$ Ahmed Mohamed Reda Ragheb $^{6} \cdot$ Mohammed Nahed Attia $^{7}$. Taghreed Sayed Meshref ${ }^{1}$}

Mai Mostafa Aly

mai_heamatology@aun.edu.eg

Marwa Ahmed Abdelhameid

Marwa.ahmed@aswu.edu.eg

Shimaa Abdalla Ahmed

Shimaa.abdallah@med.svu.edu.eg

Asmaa Salah Shaltout

AsmaaShaltout@aun.edu.eg

Alaa Eldin Abdel-Moniem alaaelmoniem@aun.edu.eg

Ahmed Mohamed Reda Ragheb ahmed.m_reda@aswu.edu.eg

Mohammed Nahed Attia

mohammed_nahed@aun.edu.eg

Taghreed Sayed Meshref

Taghreed.meshref@aun.edu.eg
1 Critical Care Unit, Department of Internal Medicine, Faculty of Medicine, Assiut University, Assiut, Egypt

2 Clinical Hematology Unit, Department of Internal Medicine, Faculty of Medicine, Assiut University, Assiut, Egypt

3 Department of Internal Medicine, Faculty of Medicine, Aswan University, Aswan, Egypt

4 Department of Internal Medicine, Faculty of Medicine, Qena University, Qena, Egypt

5 Department of Medical Microbiology \& Immunology, Faculty of Medicine, Assiut University, Assiut, Egypt

6 Department of Anesthesiology, Faculty of Medicine, Aswan University, Aswan, Egypt

7 Department of Oral and Maxillofacial Surgery, Faculty of Dentistry, Assiut University, Assiut, Egypt 